\title{
Cohesinopathy mutations disrupt the subnuclear organization of chromatin
}

Scarlett Gard, William Light, Bo Xiong, Tania Bose, Adrian J. McNairn, Bethany Harris, Brian Fleharty, Chris Seidel, Jason H. Brickner, and Jennifer L. Gerton

Vol. 187 No. 4, November 16, 2009. Pages 455-462.

During production, an error was introduced into the abstract. The correct sentence appears below:

In Saccharomyces cerevisiae, chromatin is spatially organized within the nucleus with centromeres clustering near the spindle pole body, telomeres clustering into foci at the nuclear periphery, ribosomal DNA repeats localizing within a single nucleolus, and transfer RNA (tRNA) genes present in an adjacent cluster.

The html and pdf versions of this article have been corrected. The error remains only in the print version. 